Journal of Animal and Veterinary Advances 10 (5): 593-596, 2011

ISSN: $1680-5593$

(C) Medwell Journals, 2011

\title{
Characterisation of Angiostrongylus cantonensis Isolates from China by Sequences of Internal Transcribed Spacers of Nuclear Ribosomal DNA
}

\author{
${ }^{1}$ C.Y. Liu, ${ }^{2}$ R.L. Zhang, ${ }^{3}$ M.X. Chen, ${ }^{1}$ J. Li, ${ }^{1}$ L. Ai, ${ }^{1}$ C.Y. Wu, ${ }^{4}$ X.Q. Zhu and ${ }^{1}$ R.Q. Lin \\ ${ }^{1}$ College of Veterinary Medicine, South China Agricultural University, \\ 510642 Guangzhou, Guangdong Province, China \\ ${ }^{2}$ Shenzhen Center for Disease Control and Prevention, 518020 Shenzhen, Guangdong Province, China \\ ${ }^{3}$ National Institute of Parasitic Diseases, Chinese Center for Disease Control and Prevention, \\ 200025 Shanghai, China \\ ${ }^{4}$ State Key Laboratory of Veterinary Etiological Biology, \\ Key Laboratory of Veterinary Parasitology of Gansu Province, \\ Lanzhou Veterinary Research Institute, CAAS, 730046 Lanzhou, Gansu Province, China
}

\begin{abstract}
The present study examined sequence variations in the Internal Transcribed Spacers (ITS) of nuclear ribosomal DNA (rDNA) among Angiostrongylus cantonensis isolates from Shenzhen, Qingyuan, Jiangmen and Wenzhou in China. The ITS of nuclear rDNA was amplified from individual $A$. cantonensis by Polymerase Chain Reaction (PCR) and the representative amplicons were cloned and sequenced. The length of the ITS sequences was $1593-1614$ bp for all Chinese $A$. cantonensis specimens and these sequences were composed of complete ITS-1 sequence of $712-720 \mathrm{bp}$, complete $5.8 \mathrm{~S}$ sequence of $153 \mathrm{bp}$, complete ITS-2 sequence of $633-650 \mathrm{bp}$ and partial $28 \mathrm{~S}$ sequence of $70 \mathrm{bp}$. The intra-specifc sequence variation in $A$. cantonensis was $0.1-1.0 \%$ for ITS- 1 and $0.0-1.3 \%$ for ITS-2 whereas sequence comparison revealed that the inter-specifc sequence differences were higher: $15.0-34.6 \%$ for ITS-1 and 22.7-24.2\% for ITS-2 between $A$. cantonensis and other Angiostrongylus sp. The results showed that the ITS sequences were conserved among the $A$. cantonensis isolates however, they were quite different from that of other Angiostrongylus species. Therefore, ITS sequences could provide useful genetic markers for the specific identification and genetic characterization of Angiostrongylus sp.
\end{abstract}

Key words: Angiostrongylus cantonensis, Internal Transcribed Spacer (ITS), ribosomal DNA (rDNA), Polymerase Chain Reaction (PCR), sequence analysis, China

\section{INTRODUCTION}

Angiostrongylus cantonensis is a rat lungworm usually inhabiting the pulmonary arteries and right ventricle of rats. Human is a non-permissive host becoming infected with $A$. cantonensis when they ingest the infective third-stage larvae of this parasite contained in raw or undercooked food (Thiengo et al., 2010). The larvae penetrate into the blood vessels of the human intestinal tract and eventually reach the meninges.

Most of the worms die shortly after reaching the meninges and do not develop into adults. Infection of this parasite in humans leads to eosinophilic meningitis and eosinophilic meningoencephalitis (Bouree et al., 2010; Panackel et al., 2006; Ramirez-Avila et al., 2009). The disease is endemic in Asia and some Pacifc islands. Now, the parasite has been found to infect humans and other mammals across a wide and ever-increasing territory (Prociv et al., 2000). The $A$. cantonensis has been given greater attention in both clinical and laboratory studies. Nematodes of the genus Angiostrongylus are parasites of carnivores and rodents (Ubelaker, 1986). The occurrence of such parasites in humans emphasizes the importance of a precise differentiation among different Angiostrongylus species.

The specific identification of larvae and adult worms among Angiostrongylus sp. based on morphological characters is difficult due to vague and similar descriptions in size and body shapes among different species (Robles et al., 2008; Ubelaker, 1986). Consequently, molecular methods would provide alternative approaches to characterize and identify Angiostrongylus sp. A previous study revealed that $A$. cantonensis, $A$. costaricensis and $A$. vasorum could

Corresponding Author: R.Q. Lin, Department of Parasitology, College of Veterinary Medicine, South China Agricultural University, 483 Wushan Street, Tianhe District, 510642 Guangzhou, Guangdong Province, The People's Republic of China 
be differentiated by polymerase chain reaction-restriction fragment length polymorphism (Caldeira et al., 2003). The 5 ' end of the Small Subunit (SSU) $r R N A$ gene provided a genetic marker to identify infective 3rd juvenile stage of $A$. cantonensis and distinguished it from other species (Fontanilla and Wade, 2008). The A. vasorum isolates were characterized using the mitochondrial $\mathrm{COI}$ gene and the second Internal Transcribed Spacer (ITS-2) of nuclear ribosomal DNA (rDNA) (Jefferies et al., 2009). Recently, phylogenetic relationship was re-constructed for $A$. cantonensis, $A$. costaricensis, $A$. malaysiensis and $A$. vasorum based on $C O I$ gene sequences and the results showed that the COI gene sequences might be a useful marker for differentiating geographical isolates of $A$. cantonensis and for uncovering cryptic species (Eamsobhana et al., 2010).

The Internal Transcribed Spacer (ITS-1, ITS-2) sequences have been proven to provide useful genetic markers for the accurate identification of a number of parasite groups (Li et al., 2006; Zhu et al., 2007; Lin et al., 2008). However, prior to the present study, there had been no reports characterizing $A$. cantonensis isolates from China using ITS sequences.

Therefore, the objectives of the present study were to characterize the rDNA region spanning the ITS-1, 5.8 S gene and the ITS- 2 of $A$. cantonensis isolates from China and to determine the intra-specific variation within $A$. cantonensis and the inter-specific difference among Angiostrongylus sp.

\section{MATERIALS AND METHODS}

Parasites and DNA extraction: Adult $A$. cantonensis samples were collected from the lungs of infected rats from different geographical localities in China. Details of each sample used in the study are shown in Table 1. All samples were fixed in $70 \%$ molecular grade ethanol and stored at $-20^{\circ} \mathrm{C}$. Total genomic DNA was extracted from individual worms by treatment with sodium dodecyl sulphate/proteinase $\mathrm{K}$, column-purified (Wizard ${ }^{\circledR} \mathrm{SV}$ Genomic DNA Purification System, Promega, Madison) and then eluted into $50 \mu \mathrm{L} \mathrm{H}_{2} \mathrm{O}$ according to the manufacturer's recommendations (Zhu et al., 2007). DNA was also isolated from the lung of healthy rats using the same method. DNA samples were stored at $-20^{\circ} \mathrm{C}$ until further use.

Enzymatic amplification: The rDNA region ITS plus primer flanking sequences was amplified by Polymerase Chain Reaction (PCR) using primers NC5 (forward; 5'-GTAGGTGAACCTGCGGAAGGATCATT-3') andNC2 (reverse; 5'-TTAGTTTCTTTTCCTCCGCT-3'). PCR reactions $(25 \mu \mathrm{L})$ were performed in $3 \mathrm{mM}$ of $\mathrm{MgCl}_{2}$, $0.5 \mu \mathrm{M}$ of each primer, $2.5 \mu \mathrm{L} 10 \times \mathrm{rTaq}$ buffer, $0.2 \mathrm{mM}$ of each dNTPs, $1.25 \mathrm{U}$ of rTaq DNA polymerase (TaKaRa)
Table 1: Angiostrongylus contonensis samples from China used in the present study and GenBank ${ }^{\mathrm{TM}}$ accession numbers of ITS sequences for $A$. contonensis and other Angiostrongylus species

\begin{tabular}{|c|c|c|c|c|}
\hline Species & $\begin{array}{l}\text { Sample } \\
\text { codes }\end{array}$ & Host & $\begin{array}{c}\text { Geographical } \\
\text { origin }\end{array}$ & $\begin{array}{c}\text { GenBank }^{\mathrm{TM}} \\
\text { accession No. }\end{array}$ \\
\hline \multirow[t]{13}{*}{ A. contonensis } & AcanSZ1 & Rat & Shenzhen, China & HQ540542 \\
\hline & AcanSZ2 & Rat & Shenzhen, China & HQ540543 \\
\hline & AcanSZ3 & Rat & Shenzhen, China & HQ540544 \\
\hline & AcanSZ4 & Rat & Shenzhen, China & HQ540545 \\
\hline & AcanSZ5 & Rat & Shenzhen, China & HQ540546 \\
\hline & AcanSZ6 & Rat & Shenzhen, China & HQ540547 \\
\hline & AcanQY1 & Rat & Qingyuan, China & HQ540548 \\
\hline & AcanQY2 & Rat & Qingyuan, China & HQ540549 \\
\hline & AcanJM & Rat & Jiangmen, China & HQ540550 \\
\hline & AcanWZ & Rat & Wenzhou, China & HQ540551 \\
\hline & AcanU & $\begin{array}{l}\text { Parmarion } \\
\text { martensi }\end{array}$ & Hawaii, USA & GU733322 \\
\hline & AcanB & Unknown & Brazil & GU733321 \\
\hline & AcanP & Unknown & Philippines & EU636008 \\
\hline \multirow[t]{2}{*}{ A. vasorum } & AvasG & $\begin{array}{l}\text { Canis } \\
\text { fomilioris }\end{array}$ & Germany & GU045375 \\
\hline & AvasC & $\begin{array}{l}\text { Vulpes } \\
\text { vulpes }\end{array}$ & Canada & GU045368 \\
\hline \multirow[t]{2}{*}{ A. costoricensis } & AvasU & Gastropod & United Kingdom & GU733324 \\
\hline & $A \cos B$ & Unknown & Brazil & GU587748 \\
\hline
\end{tabular}

and $1 \mu \mathrm{L}$ of DNA sample in a thermocycler (Biometra) under the following conditions: after an initial denaturation at $94^{\circ} \mathrm{C}$ for $5 \mathrm{~min}$, then $94^{\circ} \mathrm{C}$ for $30 \mathrm{sec}$ (denaturation); $50^{\circ} \mathrm{C}$ for $30 \mathrm{sec}$ (annealing); $72^{\circ} \mathrm{C}$ for $1 \mathrm{~min}$ (extension) for 35 cycles followed by a final extension at $72^{\circ} \mathrm{C}$ for $5 \mathrm{~min}$. Samples without genomic DNA were included in each PCR run as negative controls.

Also host (rat) control-DNA samples were subjected to the same amplification procedures as for parasite DNA. Each amplicon $(5 \mu \mathrm{L})$ was examined on agarose gels stained with ethidium bromide and photographed using a gel documentation system. The DL 2000 marker (TaKaRa) was utilized to estimate the sizes of the ITS PCR products.

Purification, cloning, sequencing and analysis of ITS rDNA: Representative PCR products were purified using spin columns (Agarose Gel DNA Purification Kit Ver 2.0, TaKaRa) and the purified PCR products were ligated into the pGEM-T Easy plasmid vector (Promega) according to manufacturer's recommendations. The recombinant plasmid was then transformed into JM109 competent cells (Promega) and positive transformants containing recombinant plasmids were selected by PCR amplifcation. Cell cultures with confrmed recombinant plasmid were sent to Shanghai Songon Biological Engineering Biotechnology Company for sequencing. Three colonies from each sample were sequenced from both directions. Pairwise comparisons were made of the level of sequence differences according to a method reported previously (Chilton et al., 1995).

\section{RESULTS AND DISCUSSION}

Genomic DNA was prepared from 10 individuals of A. cantonensis from Shenzhen, Qingyuan, Jiangmen and 
Table 2: Pairwise comparison of sequence differences (in\%) in the ITS-1 (above the diagonal) and ITS-2 (below the diagonal) rDNA among Angiostrongylus cantonensis isolates from China and as well as other Angiostrongylus species

\begin{tabular}{|c|c|c|c|c|c|c|c|c|c|c|c|c|c|c|c|c|c|}
\hline $\begin{array}{l}\text { Sample } \\
\text { codes }\end{array}$ & $\begin{array}{l}\text { Acan } \\
\text { SZ1 }\end{array}$ & $\begin{array}{l}\text { Acan } \\
\text { SZ2 }\end{array}$ & $\begin{array}{l}\text { Acan } \\
\text { SZ3 }\end{array}$ & $\begin{array}{l}\text { Acan } \\
\text { SZ4 }\end{array}$ & $\begin{array}{l}\text { Acan } \\
\text { SZ5 }\end{array}$ & $\begin{array}{l}\text { Acan } \\
\text { SZ6 }\end{array}$ & $\begin{array}{l}\text { Acan } \\
\text { QY1 }\end{array}$ & $\begin{array}{l}\text { Acan } \\
\text { QY2 }\end{array}$ & $\begin{array}{l}\text { Acan } \\
\text { JM }\end{array}$ & $\begin{array}{l}\text { Acan } \\
\text { WZ }\end{array}$ & $\begin{array}{c}\text { Acan } \\
\mathrm{U}\end{array}$ & $\begin{array}{c}\text { Acan } \\
\text { B }\end{array}$ & $\begin{array}{l}\text { Acan } \\
\mathrm{P}\end{array}$ & $\begin{array}{c}\text { Avas } \\
\mathrm{G}\end{array}$ & $\begin{array}{c}\text { Avas } \\
\text { C }\end{array}$ & $\begin{array}{c}\text { Avas } \\
\text { U }\end{array}$ & $\begin{array}{c}A \cos \\
\mathrm{B}\end{array}$ \\
\hline AcanSZ1 & - & 0.1 & 0.3 & 0.3 & 0.4 & 0.1 & 0.4 & 0.4 & 0.4 & 0.7 & 0.8 & 0.8 & - & 33.6 & 33.1 & 33.6 & 33.2 \\
\hline AcanSZ2 & 0.6 & - & 0.1 & 0.1 & 0.1 & 0.3 & 0.3 & 0.3 & 0.3 & 0.6 & 0.7 & 0.7 & - & 33.6 & 33.0 & 33.6 & 33.0 \\
\hline AcanSZ3 & 0.8 & 0.2 & - & 0.3 & 0.1 & 0.4 & 0.4 & 0.4 & 0.4 & 0.7 & 0.8 & 0.8 & - & 33.8 & 33.3 & 33.8 & 33.3 \\
\hline AcanSZ4 & 0.3 & 0.3 & 0.5 & - & 0.1 & 0.4 & 0.4 & 0.4 & 0.4 & 0.7 & 0.8 & 0.8 & - & 33.8 & 33.3 & 33.8 & 33.3 \\
\hline AcanSZ5 & 0.6 & 0.0 & 0.2 & 0.3 & - & 0.3 & 0.3 & 0.3 & 0.3 & 0.6 & 0.7 & 0.7 & - & 33.4 & 32.9 & 33.4 & 32.9 \\
\hline AcanSZ6 & 0.2 & 0.5 & 0.6 & 0.2 & 0.5 & - & 0.3 & 0.3 & 0.6 & 0.3 & 0.4 & 0.4 & - & 33.9 & 33.4 & 33.9 & 32.6 \\
\hline AcanQY1 & 0.6 & 0.0 & 0.2 & 0.3 & 0.0 & 0.5 & - & 0.3 & 0.6 & 0.3 & 0.4 & 0.4 & - & 33.9 & 33.4 & 33.9 & 32.6 \\
\hline AcanQY2 & 0.8 & 0.8 & 1.0 & 0.5 & 0.8 & 0.6 & 0.8 & - & 0.6 & 0.6 & 0.7 & 0.7 & - & 33.9 & 33.4 & 33.9 & 33.5 \\
\hline AcanJM & 0.6 & 0.0 & 0.2 & 0.3 & 0.0 & 0.5 & 0.0 & 0.8 & - & 0.9 & 1.0 & 1.0 & - & 33.9 & 33.4 & 33.9 & 33.8 \\
\hline AcanWZ & 0.9 & 1.0 & 1.1 & 0.2 & 1.0 & 0.8 & 1.0 & 0.5 & 1.0 & - & 0.7 & 0.7 & - & 34.4 & 33.8 & 34.4 & 32.2 \\
\hline AcanU & - & - & - & - & - & - & - & - & - & - & - & 0.1 & - & 34.6 & 34.1 & 34.6 & 34.1 \\
\hline AcanB & - & - & - & - & - & - & - & - & - & - & - & - & - & 34.5 & 33.9 & 34.5 & 33.9 \\
\hline AcanP & 1.3 & 0.8 & 1.0 & 1.0 & 0.8 & 1.1 & 0.8 & 1.1 & 0.8 & 1.3 & - & - & - & - & - & - & - \\
\hline AvasG & 22.7 & 22.7 & 22.7 & 22.7 & 22.7 & 22.7 & 22.7 & 22.7 & 22.7 & 23.2 & - & - & 22.7 & - & 0.1 & 0.1 & 15.0 \\
\hline AvasC & 23.6 & 23.6 & 23.6 & 23.6 & 23.6 & 23.6 & 23.6 & 23.6 & 23.6 & 24.2 & - & - & 23.7 & 0.6 & - & 0.1 & 15.0 \\
\hline AvasU & - & - & - & - & - & - & - & - & - & - & - & - & - & - & - & - & 15.0 \\
\hline$A \cos B$ & - & - & - & - & - & - & - & - & - & - & - & - & - & - & - & - & - \\
\hline
\end{tabular}

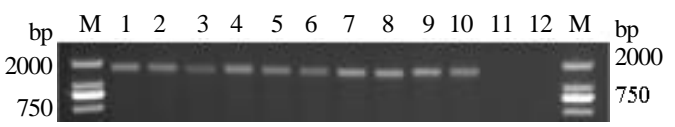

Fig. 1: Agarose gel electrophoresis of amplifed PCR products for the Internal Transcribed Spacers (ITS) of rDNA of $A$. cantonensis samples from different locations in China. Lanes 1-12 represent samples AcanSZ1, AcanSZ2, AcanSZ3, AcanSZ4, AcanSZ5, AcanSZ6, AcanQY1, AcanQY2, AcanJM, AcanWZ, host control and negative (no-DNA) control, respectively. $\mathrm{M}$ represents a DNA size marker (ordinate values bp)

Wenzhou in China (Table 1). As expected, a fragment of approximately $1600 \mathrm{bp}$ in length was amplified from each parasite gDNA. On agarose gels, there was no size variation detected among all of the amplicons and no products were amplified from the host or no-DNA control samples (Fig. 1). Representative PCR products were purified and cloned into pGEM-T Easy plasmid vector. The positive recombinant plasmids were selected by bacterial PCR amplifcation.

The obtained rDNA sequences of $A$. cantonensis isolates were 1593-1614 bp in length and their $\mathrm{A}+\mathrm{T}$ contents were $58.03-58.36 \%$. These sequences were composed of complete ITS-1 sequence of $712-720 \mathrm{bp}$ complete $5.8 \mathrm{~S}$ rDNA of $153 \mathrm{bp}$, complete ITS-2 sequence of 633-650 bp and partial $28 \mathrm{~S}$ sequence of $70 \mathrm{bp}$ (GenBank Accession numbers HQ540542-HQ540551).

The lengths and sequences of $5.8 \mathrm{~S}$ gene for all $A$. cantonensis isolates were identical whereas there was one nucleotide difference in the $5.8 \mathrm{~S} \mathrm{rDNA}$ sequences of A. cantonensis and $A$. vasorum available in GenBank ${ }^{\mathrm{TM}}$ (GU045375 and GU045368). The variation in length of the ITS-1 and ITS-2 sequences among $A$. cantonensis isolates was related to the insertions/deletions of nucleotides within the simple sequence repeats $(\mathrm{AT})_{\mathrm{n}}$. In the ITS-1 rDNA, sequence variation among $A$. cantonensis from the USA (GU733322), Brazil(GU733321) and 4A. cantonensis isolates from China was $0.1-1.0 \%$ (Table 2). However, the inter-specifc sequence differences were 15.0-34.6\% among A. cantonensis and other Angiostrongylus species (Table 2). For instance, sequence differences in the ITS-1 between $A$. cantonensis and A. vasorum (GU045375, GU045368 and GU733324) were $32.9-34.6 \%$ and between $A$. cantonensis and $A$. costaricensis (GU587748) were $32.2-34.1 \%$ (Table 2).

Variation in the simple sequence repeats $(\mathrm{AT})_{\mathrm{n}}$ of about 18 bp were detected in the ITS-1 rDNA among A. cantonensis samples. Compared with A. vasorum, A. cantonensis had deletions in the ITS-1 sequences. In the ITS-2 rDNA while the intra-specifc sequence variation among $A$. cantonensis from Philippines (EU636008) and 4 isolates from China was $0.0-1.3 \%$, the inter-specifc sequence differences between $A$. cantonensis and A. vasorum (GU045375 and GU045368) were $22.7-24.2 \%$ (Table 2). Variation in the simple sequence repeats $(\mathrm{GT})_{\mathrm{n}}$ of about $18 \mathrm{bp}$ were detected among $A$. cantonensis for ITS-2. Compared with $A$. vasorum, the $A$. cantonensis had 2 additional $(\mathrm{GT})_{\mathrm{n}}$ repeats in the ITS- 2 sequence.

\section{CONCLUSION}

The present study characterized A. cantonensis isolates from Shenzhen, Qingyuan, Jiangmen and Wenzhou in China by ITS rDNA sequences. Sequence comparison revealed that the inter-specifc sequence differences among Angiostrongylus species were significantly higher than intra-specifc sequence variations within $A$. cantonensis. There were a number of nucleotide positions in the ITS-1 and ITS-2 sequences with no apparent intra-specifc variation but distinct differences 
among $A$. cantonensis, $A$. vasorum and $A$. costaricensis which may provide useful genetic markers for the identification and differentiation of different Angiostrongylus species.

\section{ACKNOWLEDGEMENTS}

This study is supported in part by grants from the Program for Changjiang Scholars and Innovative Research Team in University (Grant No. IRT0723) and the State Key Laboratory of Veterinary Etiological Biology, Lanzhou Veterinary Research Institute, Chinese Academy of Agricultural Sciences (Grant No. SKLVEB2009KFKT0 14).

\section{REFERENCES}

Bouree, P., D. Dumazedier and N. Dahane, 2010. Angiostrongylosis or eosinophilic meningitis. Rev. Prat., 60: 456-458.

Caldeira, R.L., O.S. Carvalho, C.L. Mendonca, C. GraeffTeixeira and M.C. Silva et al., 2003. Molecular differentiation of Angiostrongylus costaricensis. $A$. cantonensis and $A$. vasorum by polymerase chain reaction-restriction fragment length polymorphism. Mem. Inst. Oswaldo. Cruz., 98: 1039-1043.

Chilton, N.B., R.B. Gasser and I. Beveridge, 1995. Differences in a ribosomal DNA sequence of morphologically indistinguishable species within the Hypodontus macropi complex (Nematoda: Strongyloidea). Int. J. Parasitol., 25: 647-651.

Eamsobhana, P., P.E. Lim, G. Solano, H. Zhang, X. Gan and H.S. Yong, 2010. Molecular differentiation of Angiostrongylus taxa (Nematoda: Angiostrongylidae) by cytochrome c oxidase subunit I (COI) gene sequences. Acta Trop., 116: 152-156.

Fontanilla, I.K. and C.M. Wade, 2008. The small subunit (SSU) ribosomal (r) RNA as a genetic marker for identifying infective 3rd juvenile stage Angiostrongylus cantonensis. Acta. Trop., 105: 181-186.

Jefferies, R., S.E. Shaw, M.E. Viney and E.R. Morgan, 2009. Angiostrongylus vasorum from South America and Europe represent distinct lineages. Parasitology, 136: $107-115$.
Li, M.W., X.Q. Zhu, R.B. Gasser, R.Q. Lin, R.A. Sani, Z.R. Lun and D.E. Jacobs, 2006. The occurrence of Toxocara malaysiensis in cats in China, confirmed by sequence-based analyses of ribosomal DNA. Parasitol. Res., 99: 554-557.

Lin, R.Q., L. Ai, F.C. Zou, J.J. Verweij and Q. Jiang et al., 2008. A multiplex PCR tool for the specific identification of Oesophagostomum spp. from pigs. Parasitol. Res., 103: 993-997.

Panackel, C.V., G. Cherian, K. Vijayakumar and R.N. Sharma, 2006. Eosinophilic meningitis due to Angiostrongylus cantonensis. Indian J. Med. Microbiol., 24: 220-221.

Prociv, P., D.M. Spratt and M.S. Carlisle, 2000. Neuro-angiostrongyliasis unresolved issues. Int. J. Parasitol., 30: 1295-1303.

Ramirez-Avila, L., S. Slome, F.L. Schuster, S. Gavali, P.M. Schantz, J. Sejvar and C.A. Glaser, 2009. Eosinophilic meningitis due to Angiostrongylus and Gnathostoma species. Clin. Infect. Dis., 48: $322-327$.

Robles, M.R., G.T. Navone and J.M. Kinsella, 2008. A new angiostrongylid (Nematoda) species from the pulmonary arteries of Akodon azarae (Rodentia: Cricetidae) in Argentina. J. Parasitol., 94: 515-519.

Thiengo, S.C., A. Maldonado, E.M. Mota, E.J. Torres and R. Caldeira et al., 2010. The giant African snail Achatina fulica as natural intermediate host of Angiostrongylus cantonensis in Pernambuco, Northeast Brazil. Acta. Trop., 115: 194-199.

Ubelaker, J.E., 1986. Systematics of species referred to the genus Angiostrongylus. J. Parasitol., 72: $237-244$.

Zhu, X.Q., S. D'Amelio, R.B. Gasser, T.B. Yang and L. Paggi et al., 2007. Practical PCR tools for the delineation of Contracaecum rudolphii $\mathrm{A}$ and Contracaecum rudolphii B (Ascaridoidea: Anisakidae) using genetic markers in nuclear ribosomal DNA. Mol. Cell. Probes, 21: 97-102. 